\title{
Rol de los Apoderados y Política de Participación del Ministerio de Educación 2002-2016. El caso de dos establecimientos educacionales de una comuna de la Región de Los Ríos*
}

\author{
Role of Guardians and Participation Policy of the Ministry of Education 2002-2016. \\ The case of two educational establishments in a commune of the Los Ríos Region
}

\author{
Rodrigo Lagos Gómez $z^{a}$, Marta Silva Fernández $z^{b, c}$ \\ ${ }^{a}$ Magister en Educación, doctorante en la Universidad Católica de Temuco, Chile. \\ profesor.rodrigo.lagos@gmail.com \\ ${ }^{b}$ PH.D. Universidad de California Santa Barbara, EE. UU. \\ ${ }^{c}$ Docente de la Universidad Austral de Chile. \\ marta.silva@uach.cl
}

\section{RESUMEN}

Este artículo presenta resultados de una investigación cualitativa que buscó dar cuenta de los procesos de participación de padres y apoderados en dos establecimientos de alta vulnerabilidad escolar de una comuna en la Región de los Ríos, Chile, en el contexto de las comunidades educativas. Se buscó establecer las formas de participación, el sentido de pertenencia, diferencias entre ambos establecimientos y los principios de gestión dados por la política ministerial. Mediante el análisis de entrevistas a directores, profesores, apoderados y estudiantes, se evidenció que las formas de participación de los padres y apoderados se adscriben a un rol tradicional utilitario donde, aun sintiéndose parte de una comunidad educativa, participar significa informarse y colaborar, entendiéndose que las formas de participación reflejan una posición subordinada, circunscrita a un rol periférico.

Palabras clave: Relación padres-escuela, participación de los padres, política educacional.

\begin{abstract}
This article presents the results of a qualitative research that sought to account for the participation processes of parents and guardians in two schools with high school vulnerability in a commune in the Los Ríos Region, Chile, in the context of educational communities. It was sought to establish the forms of participation, the sense of belonging, differences between both establishments and the management principles given by the ministerial policy. Through the analysis of interviews with principals, teachers, guardians and students, it was evidenced that the forms of participation of the parents and guardians are ascribed to a traditional utilitarian role where, even though they feel part of an educational community, participating means being informed and collaborating, understanding that the forms of participation reflect a subordinate position, circumscribed to a peripheral role.

Key words: Parent-school relationship, parental involvement, educational policy.
\end{abstract}


Estudios Pedagógicos XLVII N 3: 359-378, 2021

ROL DE LOS APODERADOS Y POLÍTICA DE PARTICIPACIÓN DEL MINISTERIO DE EDUCACIÓN 2002-2016. EL CASO DE DOS ESTABLECIMIENTOS EDUCACIONALES DE UNA COMUNA DE LA REGIÓN DE LOS RÍOS

\section{INTRODUCCIÓN}

En este artículo se presentan resultados de un estudio cualitativo realizado en dos establecimientos educacionales con alta vulnerabilidad escolar, pertenecientes a una comuna de la Región de Los Ríos en Chile. Se investigó sobre el rol de los padres y apoderados en cuanto a su participación en la escuela, y cómo se relaciona este rol con lo descrito en la Política de Participación de Padres, Madres y Apoderados/as en el Sistema Educativo del Ministerio de Educación (en adelante PPPA) que estuvo vigente de 2002 a 2016 (Ministerio de Educación, 2002; 2017). En ese contexto, para la delimitación teórica del objeto de estudio, la relación de la escuela con los padres y apoderados, se consideraron dos dimensiones: la primera, respecto de la incidencia directa de padres y apoderados en el rendimiento de los escolares y la segunda en la consideración de estos como actores relevantes de los procesos de gestión. Respecto de la primera dimensión, numerosas investigaciones expresan consistentemente que los padres son un factor clave de impacto en los logros escolares de los estudiantes y más aún en los niveles socioeconómicos bajos (Stevenson y Baker, 1987; Rodríguez 1994; Jadue, 1997; Bellei et al., 2004). Respecto de la segunda dimensión, de la gestión, hay escuelas que han implementado una serie de acciones para vincularse activamente con los padres y apoderados, sobre todo en ambientes de vulnerabilidad (Bellei et al., 2004). Sin embargo, incluso estas escuelas han mantenido la gestión de la relación con los padres y apoderados principalmente desde un rol utilitario, que se enfoca en inducir el buen comportamiento y contribuir materialmente (Bedwell, 2004; Rivera y Milicic, 2006; Gubbins, 2012).

Por lo tanto, existe una tensión dado que no hay un número significativo de investigaciones en esta temática en Chile, particularmente en la Región de los Ríos, en relación a indagar cómo es la participación de los padres y apoderados en la comunidad educativa entendiendo, de acuerdo a lo recientemente planteado, la suma importancia que tiene para los logros de aprendizaje de los y las estudiantes y cómo tendría que configurarse para mejorar la gestión educativa. En este sentido, la participación y sus formas no tienen características estándar, por lo que este estudio reveló los significados particulares de los actores del medio educativo respecto de la participación de los padres y apoderados; significados que explican las acciones que toman periódicamente acerca de ésta.

En esta línea, la revisión y análisis de la bibliografía, especialmente la referida al Ministerio de Educación, deja entrever poca claridad respecto a la existencia del sentido de pertenencia entre sus participantes, particularmente padres y apoderados. En otras palabras, este estudio también buscó develar el sentido de comunidad que ellos y ellas experimentan en tanto relaciones que implican pertenencia. En definitiva, se evidencia la necesidad de explorar sobre la participación de los padres y apoderados y el sentido de pertenencia en relación a sus llamadas comunidades educativas.

Respecto al problema planteado y basado en las entrevistas realizadas a apoderados, profesores, estudiantes y directores, se analiza la participación en base a cuatro dimensiones: 1) las formas de participación de los padres y apoderados, sus significados atribuidos por los propios apoderados, estudiantes, profesores y directores; 2) el sentido de pertenencia de los padres y apoderados respecto de la comunidad educativa y su influencia en la participación según los actores de dicha comunidad; 3) diferencias y similitudes existentes en la participación de padres y apoderados y el sentido de pertenencia en ambos establecimientos; y 4) los principios de gestión que subyacen a las formas participación y el conocimiento de la PPPA. 
La comprensión este problema de estudio requiere entonces la revisión del concepto de participación y su tratamiento en el contexto chileno en tanto política ministerial relacionada con la participación ciudadana, las comunidades educativas, el sentido de pertenencia a ellas, y la política aplicada a los establecimientos educacionales.

\section{PARTICIPACIÓN DE LOS PADRES Y APODERADOS EN EN LA ESCUELA: SU IMPACTO EN EL RENDIMIENTO ESCOLAR}

Varias investigaciones ponen de relieve la fuerte influencia de los padres en el rendimiento escolar, la cual se verifica incluso independiente de su presencia física (Rodríguez, 1994; Alcalay et al., 2003; Alcalay et al., 2005; Navarro et al., 2005; Petrelli, 2008). Esta influencia en el rendimiento se observa más acusadamente en las familias de bajo nivel socioeconómico y se refleja no sólo en las calificaciones escolares, sino que también a los resultados SIMCE (Jadue, 1997; Agencia de la Calidad de la Educación, 2015).

El Sistema de Medición de la Calidad de la Educación, (SIMCE), se inició en 1988 y es la herramienta del Ministerio de Educación que mide el logro escolar, en tanto aprendizajes logrados por los estudiantes en las diferentes áreas del currículum nacional, relacionándolos con el contexto escolar y social en que estos aprenden, utilizando como instrumentos pruebas escritas estandarizadas (Agencia de la Calidad de la Educación, 2021). Al analizar los informes de resultados, se observa una relación directa entre el nivel socioeconómico y los puntajes obtenidos. De esta forma, los estudiantes de los niveles socioeconómicos más bajos obtienen puntajes inferiores (Agencia de la Calidad de la Educación, 2015). Este fenómeno ocurre a pesar de los aumentos presupuestarios en educación que se anuncian con gran énfasis por las autoridades gubernamentales, pero que en la reducción práctica de esta brecha parecieran siempre resultar insuficientes.

Por lo tanto, si el impacto de la participación de los padres y apoderados en el rendimiento escolar es mayor en los estratos socioeconómicos bajos y si el aumento presupuestario no ha logrado cerrar las brechas de puntajes SIMCE, resulta lógico pensar que si se trata de mejorar estas cifras, los colegios con mayores índices de vulnerabilidad escolar, como los de este estudio, debieran tratar de estimular la participación y compromiso de sus padres y apoderados en la vida escolar lo más posible.

Este propósito se condice con el fin declarado de la PPPA ministerial orientada a favorecer una participación, dinámica, amplia y diversa de los padres y apoderados. Sin embargo, tradicionalmente los establecimientos educacionales no han favorecido este protagonismo, como lo revelan sucesivas investigaciones, sino que han circunscrito a los padres y apoderados al rol de inducir en sus hijos hábitos de higiene, normas y pautas de buen comportamiento (Gubbins, 2012; Rivera y Milicic, 2006), controlar que realicen sus deberes escolares en casa (Stevenson y Baker, 1987; Maso y Terrazas, 2016), concurrir a la escuela cuando se les solicite y contribuir con dinero y/o materiales (Almonacid et al., 2003; Bedwell, 2004), descuidando aspectos que inciden en la participación, como el sentido de pertenencia a una comunidad. 
Estudios Pedagógicos XLVII N 3: 359-378, 2021

ROL DE LOS APODERADOS Y POLÍTICA DE PARTICIPACIÓN DEL MINISTERIO DE EDUCACIÓN 2002-2016. EL CASO

DE DOS ESTABLECIMIENTOS EDUCACIONALES DE UNA COMUNA DE LA REGIÓN DE LOS RÍOS

\section{PARTICIPACIÓN CIUDADANA, COMUNIDAD EDUCATIVA Y POLÍTICA MINISTERIAL}

\subsection{PARTICIPACIÓN CIUDADANA Y EN EL ÁMBITO ESCOLAR}

En 2014, el informe del Programa de las Naciones Unidad para el Desarrollo (PNUD) denominado "Auditoría a la Democracia" señala que la participación ciudadana resulta esencial para la democracia y que se entiende como: "un proceso amplio que contempla el involucramiento en los asuntos públicos o colectivos a nivel comunitario o nacional, en los diversos niveles de gobierno y la sociedad en su conjunto" (p. 228). La misma publicación expresa que la participación ciudadana nacional es baja, afirmando que: "los bajos niveles de participación en Chile y la falta de espacios e instancias de involucramiento ciudadanos en los ámbitos públicos no permiten participación plena en la vida pública" (PNUD, 2014, p. 229). En la misma línea, la Organización para la Cooperación y el Desarrollo Económicos (OCDE), en una publicación sobre gobernanza de 2017, expresa que Chile es uno de los países de la región latinoamericana con una de las democracias mas consolidadas y estables, aún cuando, paradójicamente, existe un déficit participativo en la gobernanza.

Esta observación de la baja participación ciudadana en Chile no es algo nuevo, según se desprende de la medición sobre la participación social realizada por la Encuesta de Caracterización Socioeconómica Nacional (CASEN), de 2003 y 2015, que se aprecia en la siguiente tabla:

Tabla 1. Participación Social en Encuestas CASEN 2003 y 2015

\begin{tabular}{|c|c|c|c|}
\hline CASEN & $\begin{array}{c}\text { No participa en } \\
\text { organizaciones }\end{array}$ & $\begin{array}{c}\text { Participa en alguna } \\
\text { organización }\end{array}$ & $\begin{array}{c}\text { Participa en Centro de } \\
\text { Padres y Apoderados }\end{array}$ \\
\hline 2003 & $69,7 \%$ & $30,3 \%$ & $0,9 \%$ \\
\hline $2015^{* *}$ & $73,5 \%$ & $26,5 \%$ & $0,9 \%$ \\
\hline
\end{tabular}

* Porcentaje respecto del total de encuestados (participantes + no participantes).

**Considera participación en los últimos 12 meses.

Fuente: Elaboración propia basado en Jiménez de la Jara (2005) y Ministerio de Desarrollo Social (2018).

Frente a esta baja participación ciudadana, refrendada por evidencia obtenida en mediciones nacionales e internacionales, se han realizado diversos esfuerzos desde el gobierno a fin de promover esta participación, como por ejemplo la promulgación de la política que se trata en este artículo y otros cuerpos normativos como la ley 20.500, Sobre Asociaciones y Participación Ciudadana en la Gestión Pública (Ministerio Secretaría General de Gobierno, 2011).

En el ámbito escolar chileno, los espacios de participación ciudadana son los Centros de Padres y Apoderados y los Consejos Escolares, que funcionan en torno a directivas y representantes, no pudiendo participar directamente todos los padres y apoderados que deseen. Sin embargo, la institucionalidad generó una política de participación que 
considera, en principio, el involucramiento activo de todos los padres y apoderados. Es así como en el gobierno de Ricardo Lagos (2000-2006) se publicó la PPPA como parte de las orientaciones gubernamentales destinadas a fomentar la participación ciudadana, para el fortalecimiento la sociedad civil (Ministerio de Educación, 2002). En este sentido, la Ley General de Educación, actualmente refundida en el DFL 2, en su artículo $15^{\circ}$ señala específicamente que se promoverá la participación de todos los miembros de la comunidad educativa (Ministerio de Educación, 2009).

En lo referente al currículum escolar, se promulgó en 2016 la ley 20.911 que crea el Plan de Formación Ciudadana para los establecimientos educacionales, que en su artículo único, letra "ff", señala como objetivo: "Fomentar la participación de los estudiantes en temas de interés público" (Ministerio de Educación, 2016). Por su parte, el DFL 2, en su artículo 30, letra "d", expresa como uno de los objetivos de la Educación Media: “[...] valorar la participación ciudadana activa, solidaria y responsable, con conciencia de sus deberes y derechos [...]" (Ministerio de Educación, 2009). Dicho objetivo se materializa, por ejemplo, en las nuevas bases curriculares de $3^{\circ}$ y $4^{\circ}$ medio publicadas en 2019 y que incorporan la asignatura de Educación Ciudadana como obligatoria, expresando en sus propósitos formativos que "se espera que los jóvenes reflexionen, conozcan, ejerciten y evalúen diversas formas de participación ciudadana, considerando sus impactos y aportes al bien común" (Ministerio de Educación, 2019, p. 54).

Por consiguiente, los establecimientos escolares no pueden eludir la responsabilidad que les cabe en fomentar procesos participativos como parte de la experiencia de aprendizaje de sus estudiantes.

\subsection{COMUNIDAD EDUCATIVA Y SENTIDO DE PERTENENCIA}

Respecto del concepto de 'comunidad educativa', para efectos de esta investigación nos hemos centrado en el planteado por Martínez-Otero (2006) y en el propuesto por el Ministerio de Educación de acuerdo al DFL 2, puesto que ambos tienen como foco al estudiante.

Siguiendo a Martínez-Otero (2006) no existiría un solo tipo de comunidad educativa, por ejemplo, la familia es considerada una de ellas. Respecto de la comunidad educativa escolar, en tanto comunidad, debiera primar la interdependencia y el sentimiento de ser parte de una instancia amplia, donde se da y se encuentra apoyo en los demás miembros de la comunidad y en tanto educativa, estaría "integrada por un conjunto de personas interesadas corresponsablemente en la formación y posee un valor pedagógico fundamental. Los distintos estamentos, aun cuando tengan funciones diferenciales dentro de la institución, se comunican y cooperan" (Martínez-Otero, 2006, p. 52). De esta forma, la pretensión de construir una comunidad educativa exclusivamente a partir de la operación aditiva de los esfuerzos individuales será infructuosa. "En una verdadera comunidad se transita fácilmente del 'yo' al 'nosotros' o, si se prefiere, de la actuación solitaria a la solidaria" (Martínez-Otero, 2006, p. 53).

Desde el punto de vista de la institucionalidad educacional, según el artículo $9^{\circ}$ del DFL 2, la comunidad educativa ecorresponde a "una agrupación de personas que inspiradas en un propósito común integran una institución educativa" cuyo "objetivo común es contribuir a la formación y el logro de aprendizajes de todos los alumnos" para "asegurar su pleno desarrollo espiritual; ético, moral, afectivo, intelectual, artístico y físico"; este "propósito 
compartido se expresa en la adhesión al proyecto educativo del establecimiento y a sus reglas de convivencia establecidas en el reglamento interno". Acerca de quiénes componen esta comunidad, el mismo artículo dice: "la comunidad educativa está integrada por alumnos, alumnas, padres, madres y apoderados, profesionales de la educación, asistentes de la educación, equipos docentes directivos y sostenedores educacionales". (Ministerio de Educación, 2009, art. $9^{\circ}$ ). Estos integrantes tienen derechos y deberes que explicita la ley en su articulado.

Si colocamos la descripción legal ministerial a la luz de lo señalado por Martínez-Otero (2006) en el tema de comunidad, observamos algunas coincidencias como la existencia de un propósito común, centrado en el desarrollo humano y en los estudiantes como el foco de la comunidad. Pero, existen también disonancias, por ejemplo, la adhesión y/o cumplimiento de ciertos reglamentos, leyes, que explicitan derechos y deberes, lo que va en línea con la visión tradicional del rol del apoderado, que se reduce a facilitar aspectos útiles para la escuela, sin mayores cuestionamientos. Esto es más aproximado a las características de la sociedad y su contrato racionalizado, que a la red de apoyo recíproco de la comunidad.

En relación al sentido de pertenencia, éste es fundamental toda vez que se quiera comprender el fenómeno de la participación, puesto que quienes lo experimentan pueden influir directamente en los roles y acciones que asumirán, y por lo tanto, en el grado y calidad de su participación. En este contexto, Lehmann (1994) considera que una ventaja que presentarían los colegios particulares (subvencionados o pagados) respecto de los municipales, es que poseen un carácter individual o 'espíritu de colegio', esto es, un complejo simbólico definido del que se apropian los pertenecientes a la comunidad, se identifican y comprometen con él, orientando y motivando sus acciones. En contraste, los colegios municipales ofrecerían un complejo simbólico difuso y débil, ya que su guía de funcionamiento son las reglas y regulaciones de la burocracia pública, más que un 'espíritu propio', por lo tanto el sentido de pertenencia y las acciones comunes también serían débiles. Esta visión puede parecer taxativa y algo reduccionista, pero enfatiza el hecho de que si se quiere un cierto tipo de participación, es importante fomentar un cierto sentido de pertenencia a la comunidad.

\subsection{POLÍTICA DE PARTICIPACIÓN DE PADRES, MADRES Y APODERADOS/AS DEL MINISTERIO DE EDUCACIÓN}

Durante el año 2017, el Ministerio de Educación publicó una reformulación de la PPPA, tras 14 años de vigencia, de 2002 a 2016 (Ministerio de Educación, 2017). La PPPA 20022016 corresponde a un documento oficial que formó parte de la intención gubernamental de estimular la participación, declarando como objetivo general:

Generar condiciones institucionales y jurídicas que garanticen la promoción, fomento y desarrollo de la participación amplia, diversa y organizada de padres, madres y apoderados/as y de la comunidad en el sistema educativo. Esta participación se realiza en alianza con los demás actores de la comunidad educativa, con el fin de contribuir en la construcción, desarrollo y cumplimiento de su proyecto y metas educativo institucionales, en el marco de mejorar la calidad de la educación. (Ministerio de Educación, 2002, p. 49) 
Sin embargo, la participación no tiene una forma única. Esta participación "amplia, diversa y organizada" se clasificaba en cinco niveles, de menor a mayor involucramiento (Ministerio de Educación, 2002):

1. Nivel informativo: es el nivel mínimo de participación, referido a la entrega, por parte de la escuela, de información sobre: 1) el sentido de la educación para cada uno de los actores del sistema, 2) el Proyecto Educativo Institucional (PEI), planes y programas en ejecución 3) el conjunto de normas, reglas, derechos y deberes de cada uno de los actores del sistema, 4) información sobre los cambios y decisiones más importantes durante el año escolar, y 5) información a los apoderados sobre el rendimiento y conducta de su hijo.

2. Nivel colaborativo: es el nivel más común de participación referido a la colaboración de padres y apoderados en actividades como: eventos escolares; conseguir fondos para mejoramiento del inmueble; apoyar en la adquisición de conocimientos y en la disciplina de los niños

3. Nivel consultivo: la escuela o el Centro de Padres debe diseñar instancias de consulta participativa para la toma de decisiones en la escuela, evitando la imposición de alternativas predefinidas.

4. Nivel toma de decisiones en relación a objetivos, acciones y recursos: el primer subnivel implica la incorporación, con voz y voto de al menos un representante de apoderados en: mesas de negociación, consejos o comisiones (Consejos Directivos, de Profesores, Equipos de Gestión Escolar, etc.). En el siguiente subnivel, los apoderados pueden asumir responsabilidades en la gestión administrativa o pedagógica de la escuela, como programas o proyectos de innovación.

5. Nivel control de eficacia: en este nivel los apoderados toman un rol de supervisores del cumplimiento del proyecto educativo y de la gestión del establecimiento, en una función de accountability. Esto requiere haber logrado los niveles anteriores y considerar a los apoderados como interlocutores válidos en la gestión de la escuela.

En términos metodológicos, esta clasificación de niveles se relacionó con la información entregada por los entrevistados sobre la participación de apoderados, para así conocer cuán implicados se encuentran con respecto a esta clasificación oficial.

Finalmente, podemos señalar que la revisión bibliográfica realizada entregó respaldo sobre la relevancia de la participación de los padres y apoderados en el proceso educativo, aunque se encuentra habitualmente minimizada por su confinamiento en acciones subsidiarias y periféricas del quehacer escolar, como proveer materiales, controlar disciplina y cumplimiento de tareas. Esta escasa participación dentro de los márgenes posibles encuentra su correlato a nivel país, en que tanto encuestas nacionales como organismos internacionales expresan que la participación ciudadana en Chile está muy por debajo de lo que sería deseable. Frente a este panorama se han promulgado cuerpos normativos que respalden y fomenten mayores niveles de participación, como la PPPA. Sin embargo, el que los apoderados se involucren en estos procesos, al menos dentro de las posibilidades descritas por dicha política, no depende sólo de la existencia de documentos normativos, sino también que sean conocidos, validados y apropiados por las comunidades educativas 
a quienes están dirigidos; con sus significados atribuidos, el sentido de pertenencia a la comunidad, y cómo esta se organiza localmente; aspectos que no están estudiados exhaustivamente. Ante este vacío de información, específicamente en la región de Los Ríos, es que se realiza esta investigación en dos escuelas de la región, con el método y los resultados que se presentan a continuación.

\section{MÉTODO}

\subsection{TIPO DE ESTUDIO Y METODOLOGÍA}

El estudio se inscribe en el paradigma interpretativo, conocido también como paradigma fenomenológico, centrándose en la experiencia individual y subjetiva de los participantes en lugar de buscar generalizaciones (Latorre et al., 1996), por lo tanto se utilizaron métodos cualitativos (Denzin y Lincoln, 2011) donde se recogieron datos descriptivos, las propias palabras de los participantes (Taylor y Bodgan, 1987). La elección de este diseño cualitativo del tipo descriptivo-interpretativo, permitió analizar descriptivamente los significados del fenómeno de la participación de los padres y apoderados desde la perspectiva de los propios actores, profesores, directivos y estudiantes. La participación entonces, se entiende como una experiencia compartida, cuyo análisis descriptivo permite "interpretar los procesos y estructuras sociales” (Gurdián-Fernández, 2007, p. 152). Por lo tanto, la finalidad principal es la comprensión e interpretación de la realidad y los significados de los participantes, y no verificar teorías ni enunciar leyes de aplicación general en el ámbito educativo.

La recolección de datos se realizó mediante el diseño de una entrevista semi-estructurada debido a que permite a los participantes expresar su propia perspectiva y palabras (Kvale, 2011), manteniendo la coherencia con el tipo de estudio, ahondando en la experiencia de los participantes, lo que sirvió de insumo para su posterior análisis y discusión.

\subsection{SELECCIÓN DE ESTABLECIMIENTOS Y PARTICIPANTES}

Dado que la finalidad principal del diseño de la investigación es la comprensión e interpretación de la realidad y los significados de los participantes, no se realizó definición de población, ni determinación de tamaño de muestra siguiendo métodos estadísticos probabilísticos, ya que no se busca lograr una representación generalizable.

En cuanto a los criterios teórico-metodológicos para la selección de establecimientos, dado que se pretendía realizar una descripción profunda respecto de la participación de padres y apoderados, la cual tiene mayor impacto en sectores socioeconómicos bajos, se efectuó una aproximación a centros educativos con un índice de vulnerabilidad escolar superior al 70\%. En uno de ellos emergió la percepción de los apoderados como "muy participativos" y en otro, la percepción de "poco participativos", asimetría que ofrecía la oportunidad de profundizar en esas posibles diferencias y realizar una comparación respecto de aquellas. Adicionalmente, ambos establecimientos mostraron disponibilidad de tiempo y organización para aplicar el instrumento de investigación.

Respecto de los criterios éticos, se obtuvo autorización del sostenedor de los establecimientos y se estableció una relación de confianza con los centros educativos en los acercamientos realizados. Se presentaron los fines del estudio así como los consentimientos 
informados, que ofrecían detalles de la investigación y la debida reserva de identidad en la información entregada.

En consecuencia, el estudio se realizó en dos establecimientos educacionales de una comuna de la Región de los Ríos, ambos con un índice de vulnerabilidad superior al 70\%. El establecimiento donde inicialmente se percibía a los apoderados como "muy participativos", se denomina en este artículo como Establecimiento 1. El otro centro educativo, donde se percibía a los apoderados como "poco participativos", se denomina Establecimiento 2.

Respecto de la forma de selección de los entrevistados, siguiendo a Rodríguez et al. (1999), se empleó una selección deliberada considerando el valor de cada participante como fuente de información para el cumplimiento de los objetivos de esta investigación, con los siguientes criterios de inclusión:

- Directores: al menos un año completo de permanencia en el establecimiento.

- Profesores: al menos un año completo de permanencia en el establecimiento y jefatura de curso en segundo ciclo básico, pues la jefatura implica un conocimiento más profundo de las conductas de los apoderados en su relación con el establecimiento.

- Apoderados: ser o haber sido apoderados de primer y segundo ciclo básico, lo que le entrega experiencia de participación en más de un curso. Además, haber asistido al menos a la mitad de las reuniones convocadas el año anterior, con el fin de asegurar que han experimentado la dinámica de un período escolar completo.

- Estudiantes: pertenecer al segundo ciclo básico, lo que conlleva una edad en que sus experiencias les permitieran responder la entrevista.

El total de entrevitados ascendió a 16 personas: director, dos profesores, tres apoderados y dos estudiantes, por cada establecimiento.

\subsection{INSTRUMENTO, PRODUCCIÓN Y ANÁLISIS DE LA INFORMACIÓN}

Respecto al instrumento, se elaboró un protocolo de entrevista con preguntas semiestructuradas que cubriesen los temas clave pertinentes al estudio, los cuales fueron: 1) la forma en que participan los padres y apoderados en el establecimiento, 2) los significados que se le atribuyen a participar, 3) el sentido de pertenencia a la comunidad, y 4) los principios de gestión que subyacen a las formas de participación y conocimiento de la PPPA. Todas las personas contactadas en forma inicial para solicitar su participación, accedieron a ser entrevistadas. La toma de datos se realizó en 2015 tras la obtención de los consentimientos informados, en visitas sucesivas y con registro de voz de los informantes. Todos los participantes manifestaron buena disposición para responder las preguntas del protocolo, sin evasivas, aunque en algunos casos se pidió que respondieran con mayor detalle, para minimizar la ocurrencia de respuestas escuetas. La producción de información se realizó mediante la aplicación del protocolo de entrevista, cuyos audios se transcribieron íntegramente, exceptuando referencias que pudieran ayudar a identificar al informante usando pseudónimos para este fin. A continuación se procedió a la lectura reiterada de las entrevistas, en un proceso de familiarización y reconocimiento de patrones comunes en los testimonios. La siguiente etapa fue la construcción de las categorías de análisis y sus códigos asociados, presentándose categorías preestablecidas que apuntaban a responder 
las preguntas de investigación, así como la emergencia de categorías sobre aspectos no previstos y que aportaban puntos de análisis importantes. Este proceso de análisis se realizó sin uso de software especializado, debido a que el volumen de las entrevistas no lo ameritaba, dejando como ventaja la familiarización y el comienzo de análisis inmediato. Finalmente se realizó el volcado del análisis en papel, presentando tablas resumen de categorías y códigos para facilitar la lectura, comparación y obtención de conclusiones.

En cuanto a los aprendizajes del proceso, sobresalió la buena disposición de los informantes a emitir sus opiniones abiertamente, lo que revela la necesidad de ser consultados y oídos en sus propias palabras respecto del contexto en que están inmersos. En la fase de análisis de las entrevistas se reveló cuán profundamente influyen en la conducta los significados atribuidos por las personas, tal como se describe en la revisión bibliográfica y cómo a través del discurso se revelan estos significados.

\section{RESULTADOS Y DISCUSIÓN}

Los resultados se expresan para cada pregunta de investigación que se realizó sobre la participación de los apoderados en ambos establecimientos y su correspondencia con la PPPA ministerial. La relación de estos elementos se representa gráficamente en la siguiente figura:

Figura 1. Representación de los elementos de la investigación.

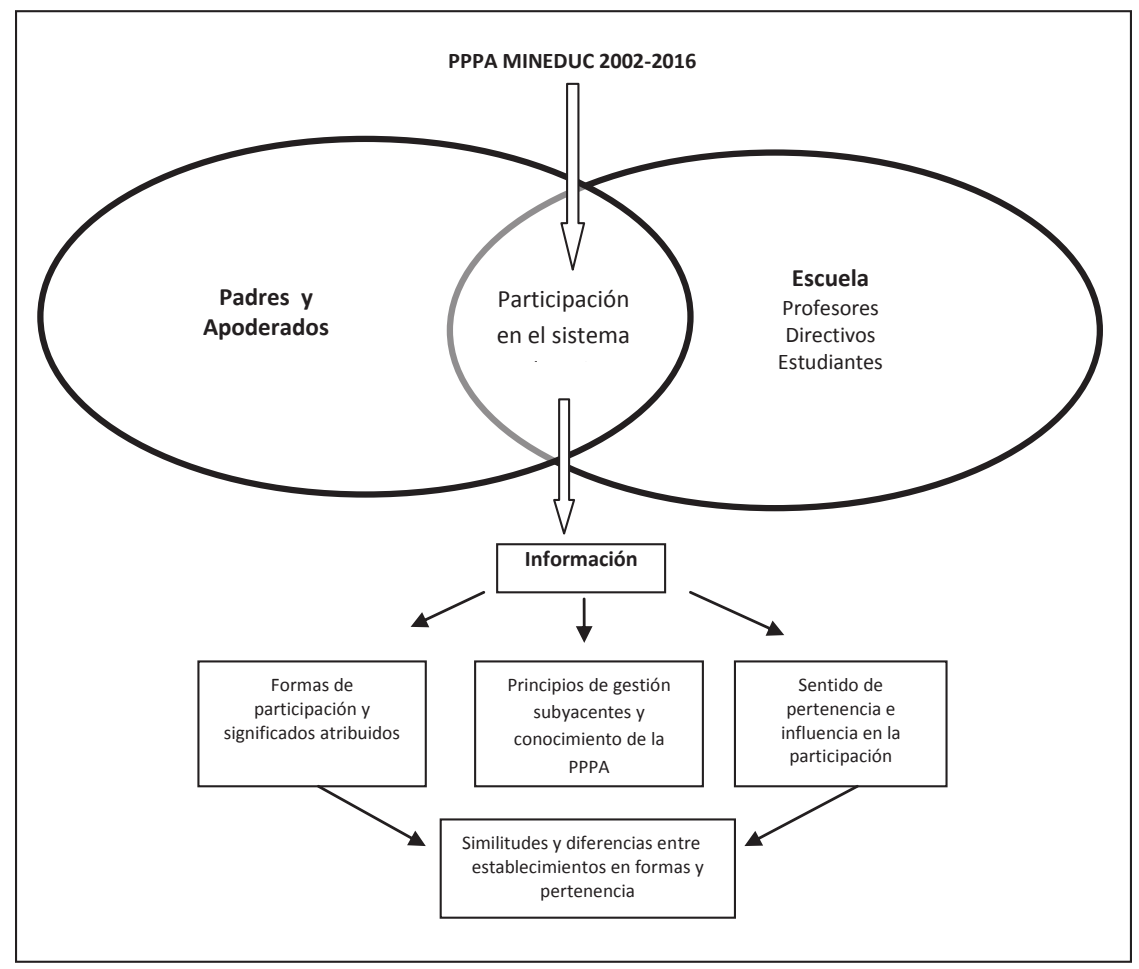


A continuación, se describen los resultados obtenidos para las preguntas una vez procesada la información, con citas textuales de los entrevistados y su correspondiente discusión. Estas citas están en letra cursiva. Las intervenciones del entrevistador están destacadas en negrita.

\subsection{FORMAS DE PARTICIPACIÓN DE PADRES Y APODERADOS, Y SUS SIGNIFICADOS ATRIBUIDOS}

Las entrevistas revelaron que las formas de participación en ambos establecimientos son reuniones, eventos de celebración o para beneficio económico y citaciones específicas a apoderados. Estas instancias se traducen en recibir información sobre aspectos administrativos, como el reglamento de convivencia, PEI, información específica sobre rendimiento o conducta de sus niños, colaborar en convivencias y actos diversos de celebración. Por lo tanto, al apoderado le compete estar atento al control de deberes escolares y en general estar a disposición de concurrir cuando las necesidades del establecimiento lo determinen, como lo evidencian las siguientes citas:

Ellos [los padres y apoderados] en primer lugar participan del proceso de sus hijos, enterándose que está pasando con ellos, asistiendo a reuniones, asistiendo a citaciones de los profesores... cuando hacemos show o una celebración, una ceremonia, ellos participan en esa ceremonia, en los show cuando se hace algún acto, el día del alumno, ellos participan.( Director Establecimiento 1)

Participamos en reuniones, haciendo cosas de, para beneficio de los niños, todo eso. También en actos, se participa de hartas cosas... también hacemos venta de empanadas, hacemos completos. (Apoderado 3 Establecimiento1)

En reuniones de apoderados que son obligatorias, eventos que se hacen, actos, día de la Madre, día del Padre, día del apoderado, aniversarios. Eso. (Profesor 1 Establecimiento 1)

Ellos participan por ejemplo cuando necesitan juntar dinero para el curso, ellos participan vendiendo cosas. [...] Y dando opiniones a los profesores, participan de reuniones y de la reunión del Centro de Padres. [...] por ejemplo para el día del niño, ellos vinieron acá a ayudar a los profesores, a hacer una pequeña convivencia a los niños. (Estudiante 1 Establecimiento 2)

Esta semblanza general es coincidente con los hallazgos de las investigaciones revisadas sobre la relación entre los padres y apoderados con los establecimientos educacionales (Almonacid et al. 2003; Bedwell, 2004; Rivera y Milicic, 2006; Gubbins, 2012) que se sintetiza en un rol auxiliar de control de tareas, disciplina y contribución material, alejado de la gestión relevante del establecimiento; en pocas palabras asumiendo una posición subordinada, lo que se aleja de la idea pregonada por el DFL 2 y la PPPA de constituir una auténtica comunidad educativa, ya que esa subordinación permanente atenta contra fundamentos comunitarios como la interdependencia de sus miembros y la acción solidaria en pos de objetivos comunes.

Respecto de los significado atribuidos, observamos que el significado de "participación" se asimila con el de "asistencia": el apoderado que participa es, esencialmente apoderado 
que asiste. Además, se observó que participación también tiene un significado de responsabilidad parental, según se desprende de las siguientes citas respecto del ideal de apoderado participativo:

Tendría que venir siempre a reunión, estar preocupado de llamar al profesor, está preocupado de saber si su hijo tiene alguna prueba, porque a uno le dan calendario, estar preocupado constantemente, en la reunión ser participativo, tratar que no sólo la directiva pueda conformar algo para el curso, si no que digamos que todos pueden aportar alguna opinión. (Apoderado 1 Establecimiento 1)

Nosotros queremos un apoderado que sea participativo, que nos venga a preguntar cosas, que esté ojalá las mayores veces, de los días, acá en el colegio consultando sobre su niño y apoyándolo. (Director Establecimiento 2)

Tiene que ser un apoderado que sea responsable de principio a fin, en qué sentido, en que ese apoderado que llegue en marzo a dejar a su niño, y durante el año, al menos una vez por semana, esté preguntando cómo va, en rendimiento, en comportamiento que son las, digamos, lo que nosotros trabajamos con los niños. (Profesor 1 Establecimiento 2)

Si el profesor pide algo siempre están ellos ahí apoyando, siempre participan las reuniones y si pasa algo ellos están ahí apoyando. (Estudiante 1 Establecimiento 2)

Así, estos dos significados preeminentes, de asistencia y responsabilidad, marcan la referencia en el significado de participación. El apoderado que participa es el que asiste, siempre dentro del rol subordinado y con ello cumpliría con su responsabilidad parental. Por tanto, por extensión, el apoderado que no asiste expresaría desinterés por sus hijos, idea con asidero ya que de los testimonios se infiere que los establecimientos no manejan definiciones ni estrategias claras de qué es participación. No obstante, adhieren en general al arquetipo del apoderado participativo que se reveló en esta investigación, que está 'en todo' y dispuesto siempre a colaborar en lo que sea necesario.

\subsection{SENTIDO DE PERTENENCIA DE LOS PADRES Y APODERADOS A LA COMUNIDAD EDUCATIVA Y SU INFLUENCIA EN LA PARTICIPACIÓN}

Entre ambos establecimientos hay opiniones divididas entre los entrevistados respecto de la relación pertenencia - participación, con los matices y diferencias que se expresan en las siguientes citas, comenzado con el Establecimiento 1:

Sí, porque siempre nos están pidiendo opiniones y cosas, yo me siento parte, porque consultan si estamos de acuerdo con lo que vamos a hacer, o lo que se va a hacer en el colegio [...] si no me sintiera parte participaría menos, no estaría ni ahí con lo del colegio, con lo que hagan acá. (Apoderado 2 Establemiento 1)

[Se sienten] muy pertenecientes y uno lo ve en los actos, por ejemplo para el Día de la Madre, se calculó que iban a venir 150 mamás y llegan 300 mamás [...] si ellos se sienten tomados en cuenta, participan, se sienten comprometidos y eso también se 
ve con el traer niños al colegio porque ahora nos ha aumentado cualquier cantidad la matrícula. (Profesor 1 Establecimiento 1)

Sí, ellos siempre dicen mi escuela, ellos se sienten con derecho de exigir cosas, incluso a tal extremo que están al tanto, como ahora todo está disponible en línea, de lo que la escuela tiene en dinero por SEP [...] Sí, participa más.

Entonces si el apoderado se siente parte, participa más ¿y si no se siente parte?

Para mí, va a participar lo justo y necesario, cuando le digan esto, hay que hacer esto, nada más. (Director Establecimiento 1)

En este punto vale citar a Giménez (1997), quien puntualiza que el sentido de pertenencia implica la inserción de una personalidad individual en una colectividad, hacia la cual se experimenta un sentimiento de lealtad y donde se asume un rol. Se observa en los testimonios que se mantiene el apego al rol tradicional abordado con detalle en el punto anterior y una participación enmarcada en la información y la colaboración, que son los niveles inferiores de la PPPA.

En el caso de los estudiantes de este establecimiento, se revelaron circunstancias personales de sus apoderados que tienen influencia en la pertenencia y la participación:

Sí, porque la mayoría de mis hermanos estudiaron aquí y que ella se siente unida al colegio [..]

Si ella no se sintiera parte de la escuela ¿participaría igual, más o menos?

Participaría menos. (Estudiante 2 Establecimiento 1)

Así, la pertenencia tiene una raigambre de historia personal, donde aflora la "lealtad" que señala Giménez (1997) y donde, en este caso, se presenta la atribución de relación interdependiente entre pertenencia y participación, refrendada por el testimonio de otro estudiante, donde además vuelve a aflorar la vinculación de participación con asistencia:

Es que en realidad como no puede venir tanto a la escuela, yo creo que [se siente perteneciente] mas o menos nomás [...]

Si su trabajo no la limitara, ¿ella participaría más?

\section{Sí. (Estudiante 1 Establecimiento 1)}

En el caso del Establecimiento 2 las opiniones presentan una mayor dispersión y se revela la creencia, por parte del director y el profesorado entrevistado, que hay una proporción de apoderados que experimentan escasa o ninguna pertenencia:

De este el punto de vista de todo lo que uno percibe, hay apoderados muy parte del colegio como hay otros apoderados que se sienten muy lejanos [...] el apoderado que no se siente parte de la escuela y que tiene un cierto compromiso con su hijo, el nieto o lo que sea, llega solamente hasta el hecho de mandarlo al colegio y cumplir con que el niño llegue la escuela [...] En cambio el apoderado que sí se siente parte de la escuela se involucra mucho más allá de esto. (Director Establecimiento 2) 
Estudios Pedagógicos XLVII N 3: 359-378, 2021

ROL DE LOS APODERADOS Y POLÍTICA DE PARTICIPACIÓN DEL MINISTERIO DE EDUCACIÓN 2002-2016. EL CASO DE DOS ESTABLECIMIENTOS EDUCACIONALES DE UNA COMUNA DE LA REGIÓN DE LOS RÍOS

Pienso que ese es el problema, que ellos no se sienten identificados con el colegio. Incluso a veces yo digo, porqué tienen a su hijo acá, si no se identifican con la escuela. [...] pienso que es directamente proporcional, por esa razón ellos no participan no más, no se identifican con la escuela. (Profesor 2 Establecimiento 2)

En el caso de los apoderados, manifiestan pertenencia ya sea por vínculos propios o a través de sus hijos. Emerge nuevamente la responsabilidad parental como motor de la participación, que puede operar independiente de la pertenencia:

Me gusta cómo trabajan los profesores acá con los niños, y estudié en este colegio, entonces es como un sentimiento que tengo con este colegio, viene de antes y me siento partícipe siempre de las cosas que están acá en este colegio [...]

Si no se sintiera parte, ¿participaría igual?

Sí, por una cosa de responsabilidad, del hecho de tener mis hijos acá. (Apoderado 1 Establecimiento 2)

Sí [se siente parte] porque yo todo lo que tengan que ver con mis hijas, yo siempre participo en las cosas del colegio [...] Yo hallo que si se siente parte [el apoderado] participa más” (Apoderado 3 Establecimiento 2)

Respecto de los estudiantes, coinciden con sus pares del Establecimiento 1. Existen circunstancias personales que influyen en la pertenencia, y que existen ciertas limitaciones prácticas como el tiempo disponible. Además, aparece nuevamente el elemento de responsabilidad parental:

Sí yo creo que sí [se siente perteneciente], porque si hay por ejemplo algún trabajo del colegio, igual lo mandan a hacer a ella. [Apoderado tiene taller de costura]

¿Crees tú que el apoderado que se siente parte del colegio participa más que el que no se siente parte?

No, yo creo que participan igual.

Entonces ¿la participación tendría que ver con una responsabilidad más que con sentimiento?

Sí, con responsabilidad más que lo del sentimiento. (Estudiante 1 Establecimiento 2)

Yo creo que sí [se siente perteneciente], porque ha tenido a todos nuestros hermanos aqui en la escuela [...] se siente parte, pero no le cabe el tiempo para participar. (Estudiante 2 Establecimiento 2)

Es de hacer notar que la pertenencia y la participación se mantienen circunscritas al rol, tradicional utilitario del apoderado. Queda en evidencia en el caso del estudiante 1 del Establecimiento 2, que señala como evidencia de la pertenencia de su apoderado el que realice trabajos de costura para el establecimiento. La emergencia de la responsabilidad parental como motor de la participación se alinea con lo descrito en el punto anterior, estar disponible para cumplir con lo que necesite el establecimiento, pudiendo ser un factor que influya en la participación con independencia de la pertenencia. 


\subsection{DIFERENCIAS Y SIMILITUDES EN LA PARTICIPACIÓN Y EL SENTIDO DE PERTENENCIA EN AMBOS ESTABLECIMIENTOS}

Según lo especificado en los dos puntos anteriores, se verifica la existencia de similitudes y diferencias entre ambos establecimentos, que se presentan en la siguiente tabla comparativa considerando los testimonios previamente citados, que se omiten para evitar redundancia.

Tabla 2. Similitudes y diferencias entre establecimientos, respecto de la participación y sentido de pertenencia

\begin{tabular}{|c|c|c|}
\hline & Similitudes & Diferencias \\
\hline $\begin{array}{c}\text { Formas de } \\
\text { participación } \\
\text { de los padres } \\
\text { y apoderados } \\
\text { y significado } \\
\text { atribuido }\end{array}$ & $\begin{array}{l}\text { Las formas de participación se } \\
\text { circunscriben al rol tradicional descrito } \\
\text { en la revisión bibliográfica: reuniones } \\
\text { informativas, celebraciones, control del } \\
\text { rendimiento y comportamiento del niño, } \\
\text { asistir cuando se le pida; en suma una } \\
\text { actitud subsidiaria a los fines del } \\
\text { establecimiento, alejada de la gestión } \\
\text { relevante. } \\
\text { Los significados atribuidos principales } \\
\text { fueron el de asistencia y el de } \\
\text { responsabilidad parental. El apoderado } \\
\text { participativo es el que asiste, el que está } \\
\text { siempre. Y además, participar es una } \\
\text { responsabilidad parental. No se evidencia } \\
\text { la participación como significado de un } \\
\text { ejercicio de ciudadanía. }\end{array}$ & $\begin{array}{l}\text { No se evidenciaron diferencias } \\
\text { destacables en las formas de } \\
\text { participación, ni en los significados. }\end{array}$ \\
\hline $\begin{array}{l}\text { Pertenencia y } \\
\text { participación }\end{array}$ & 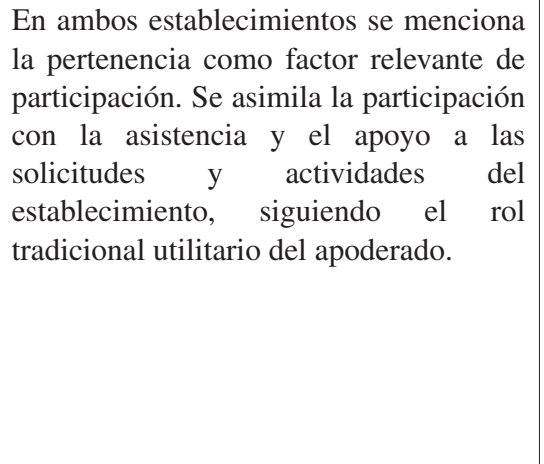 & $\begin{array}{l}\text { Establecimiento 1: La pertenencia } \\
\text { influye mayoritariamente en la } \\
\text { participación de manera directamente } \\
\text { proporcional. No se resalta baja } \\
\text { pertenencia en apoderados. } \\
\text { Establecimiento } 2 \text { : Si bien se estima } \\
\text { importante la pertenencia, la } \\
\text { responsabilidad parental emerge } \\
\text { como un factor relevante de la } \\
\text { participación. Se resalta por parte del } \\
\text { director y profesores una baja } \\
\text { pertenencia en apoderados. }\end{array}$ \\
\hline
\end{tabular}




\subsection{PRINCIPIOS DE GESTIÓN SUBYACENTES Y CONOCIMIENTO DE LA PPPA}

En cuanto a los principios de gestión que se revelan a partir de las formas de participación descritas, se evidencia una relación asimétrica entre la escuela y los padres y apoderados, en que éstos ocupan una posición subordinada, circunscritos en su participación a un rol periférico que consiste en auxiliar las solicitudes del establecimiento, controlar los deberes escolares, la disciplina, contribuir materialmente y concurrir cuando se le cita, en clara correspondencia con la evidencia aportada por las investigaciones citadas en la revisión bibliográfica. Esta situación mantiene a los apoderados alejados de la posibilidad de ejercer una incidencia sustantiva en la marcha de las instituciones educativas.

Este tipo de participación, informativa y colaborativa, se inscribe en los dos niveles inferiores de la PPPA, quedando sin efecto práctico los tres niveles superiores de participación (Ministerio de Educación, 2002). Hay que destacar que la PPPA resultó desconocida en tanto documento específico para directores, profesores y apoderados, quienes fueron consultados sobre tener conocimiento de alguna indicación específica del Ministerio de Educación sobre la participación de apoderados; respondiendo directamente que no, o hicieron alusión a generalidades que no señalan la existencia de la PPPA:

\section{Hum, eh, no. (Apoderado 1 Establecimiento 2)}

A ver, la verdad es que lo que yo he visto ahora...más que nada el Ministerio lo que hace es tratar de mantener al día al apoderado, pero más que nada, por ejemplo en el resultado de evaluaciones, por ejemplo el SIMCE. (Profesor 2 Establecimiento 1)

El Ministerio, la normativa que tiene con respecto a esto, es la participación de un adulto para que esté informado acerca de lo que sea conducta, rendimiento, o sea todo lo que tiene que ver con el quehacer educativo de un estudiante. Ellos lo piden, la legalidad dice que tiene que haber un representante pero tampoco pone por ejemplo una exigencia que diga usted tiene que asistir por lo menos una cierta cantidad o un porcentaje a las reuniones. Eso es independiente de cada colegio, las exigencias dependen de cada escuela. (Director establecimiento 2)

Este desconocimiento de la política ministerial deja al arbitrio de cada establecimiento qué es y que implica participar, lo que contribuye a que la participación sea un concepto fluido, donde cada actor interpreta y actúa según sus significados personales, lo que es poco deseable si se quiere lograr la identificación y vínculo personal de todos los apoderados con un solo complejo simbólico que lleve a un sólido compromiso comunitario. Así, los beneficios de la participación de apoderados para el rendimiento escolar y de gestión del establecimiento, que se revisaron en los antecedentes bibliográficos presentados, se ven desperdigados y debilitados, reducidos a las instancias reglamentarias en que participa regularmente sólo una pequeña fracción como las directivas de curso, el Centro de Padres y Consejo Escolar, dejando al resto de los apoderados a disposición de convocatorias eventuales como reuniones informativas y celebraciones, dejando en la superficialidad lo que especifica el DFL 2 en su artículo $15^{\circ}$, respecto de la responsabilidad de los establecimiento de promover participación de todos los miembros de la comunidad (Ministerio de Educación, 2009). 
Por lo tanto, es una política nacional que, en el contexto estudiado, se encontró inoperante en su vocación de inclusión por desconocimiento, lo que es un fallo severo considerando que su vigencia se extendió 14 años. Esto refleja también que la participación de apoderados no es una prioridad ministerial, dado que al parecer hubo escasa o nula difusión de su propia política, indicando que no se hace vínculo real entre la participación y el ejercicio de una ciudadanía involucrada, lo que lleva a una democracia en que la sociedad civil se valora esencialmente en cuanto a votos y poco más.

\section{CONCLUSIONES}

En lo que refiere a las formas de participación de los padres y apoderados, estas corresponden a reuniones, eventos de celebración o para beneficio económico y citaciones específicas a apoderados. Estas instancias se traducen en recibir información sobre aspectos administrativos, como el reglamento de convivencia, PEI, información específica sobre rendimiento o conducta de sus niños, colaborar en convivencias y actos diversos de celebración. Estas formas corresponden a las del rol tradicional del apoderado, como subsidiario de las necesidades del establecimiento y en una posición subordinada.

Por otro lado, en lo que corresponde a los significados atribuídos, hay una fuerte asimilación entre participación y asistencia. El apoderado que participa es el que asiste al establecimiento frecuentemente, por voluntad propia y cuando es convocado, dispuesto a colaborar en lo que necesite. Además, se reveló que la participación puede ser considerada como expresión de responsabilidad parental, con cierta independencia del sentido de pertenencia.

Respecto del sentido de pertenencia y la influencia en la participación, en ambos establecimientos se considera como relevante la pertenencia, en tanto factor de participación, ofreciendo varios testimonios la apreciación que la pertenencia influye sobre la participación en forma proporcional: a mayor pertenencia, mayor participación y viceversa. Sin embargo, emergieron ciertos matices en cuanto a considerar secundaria la pertenencia como factor clave de participación, primando el sentido de responsabilidad parental que se reflejaría al participar activamente, lo que significa estar a disposición de lo que se indique desde la dirección y el profesorado.

En cuanto a diferencias y similitudes entre ambos establecimientos, se observó que en formas de participación y significados atribuídos coincidieron en la relación con los padres y apoderados de la manera tradicional, utilitaria y subordinada, con asociación de la participación con asistencia y responsabilidad parental, sin diferencias destacables. En lo que corresponde a sentido de pertenencia y su influencia en la participación, en ambos establecimientos se encontraron testimonios que consideran que la pertenencia tiene directa relación con la participación. Como diferencia en este ámbito, se observa que en el Establecimiento 2 emergen referencias a la existencia de una fracción de los apoderados que experimentarían una pertenencia escasa y una mayor consideración a la responsabilidad parental como factor clave de la participación.

En lo relativo a los principios de gestión subyacentes y su relación con los niveles de participación de la PPPA del Ministerio de Educación que se encontraba vigente en el momento de realizar el levantamiento de la información, se reafirma lo que se ha expuesto en este artículo respecto a que las formas de participación reflejan una gestión de la relación de los padres 
y apoderados desde una posición subordinada, circunscritos a un rol periférico de auxiliar las necesidades del establecimiento, controlar los deberes escolares y el comportamiento, en clara correlación con la evidencia aportada por las investigaciones nacionales citadas en la revisión bibliográfica; rol que los mantiene alejados de la posibilidad de tener una incidencia sustantiva en la gestión educacional, dado que se corresponde a los dos niveles inferiores de la PPPA, quedando sin efecto práctico los tres niveles superiores de participación.

Por último, resaltar que la PPPA resultaba desconocida en tanto documento específico para los entrevistados lo que contribuye a que la participación sea 'tierra de nadie' donde cada establecimiento, incluso cada profesor, interpreta y actúa según sus significados personales, lo que implica que no se pueda avanzar en la consolidación de una gestión sistematizada en este ámbito, y, además, la ausencia de un modelo de participación ciudadana para los estudiantes. Esta ausencia de modelo implica que en su vida adulta probablemente seguirán anclados en el rol tradicional y por extensión, probablemente se mantendrá la baja participación ciudadana que ya caracteriza a nuestra sociedad, como lo indican los documentos de la OCDE (2017) y el PNUD (2014). Por lo tanto, es de esperar que la reformulación de la PPPA realizada en 2017 tenga mayor difusión en los establecimientos de Chile, a fin de proveer modelos de participación más activos para nuestros niños y jóvenes, que sean una instancia de formación que beneficie al conjunto de nuestra sociedad y de una futura vida política ciudadana que rompa el utilitarismo de manera transversal.

\section{REFERENCIAS BIBLIOGRÁFICAS}

Agencia de la Calidad de la Educación. (2021). SIMCE. Recuperado de https://www.agenciaeducacion. $\mathrm{cl} /$ simce/

Agencia de la Calidad de la Educación. (2015). Evolución de las brechas socioeconómicas de rendimiento en pruebas SIMCE. Recuperado de http://archivos.agenciaeducacion.cl/estudios/ Evolucion_brechas_socioeconomicas_de_rendimiento_en_pruebas_Simce.pdf

Alcalay, L. Flores, A. Milicic, N. Portales, J. y Torretti, A. (2003). Familia y Escuela. ¿Una Alianza Posible? Una mirada desde la Perspectiva de los Estudiantes. Psykhe, 12(2), 101-110. Recuperado de http://ojs.uc.cl/index.php/psykhe/article/view/20443

Alcalay, L. Milicic, N. y Torretti, A. (2005). Alianza Efectiva Familia-Escuela. Un Programa Audiovisual para Padres. Psykhe, 12(2), 149-161. Recuperado de https://dx.doi.org/10.4067/ S0718-22282005000200012

Almonacid, L. Herrera, B. Morales, M. y Salazar, J. (2003). Valoración de la Participación en la Escuela, desde la Perspectiva Padres-Apoderados y Docentes, del Establecimiento Municipal, Caupolicán de Temuco, Período Escolar 2003. Tesis de grado, Facultad de Educación, Universidad Católica de Temuco. Temuco.

Bedwell, G. (2004). Participación de los Padres, Madres y Apoderados en el Ámbito Educativo. Memoria de título, Facultad de Ciencias Sociales, Universidad de Chile. Santiago de Chile.

Bellei, C. Muñoz, G. Pérez, L. y Raczynski, D. (2004). ¿Quién dijo que no se puede? Escuelas Efectivas en Sectores de Pobreza. Santiago de Chile: UNICEF. Recuperado de http://www. unicef.cl/centrodoc/escuelas_efectivas/escuela\%20efectivas.pdf

Denzin, N. y Lincoln, Y. (2011). Manual de Investigación Cualitativa Volumen I. El campo de la investigación cualitativa. Barcelona: Editorial Gedisa.

Giménez, G. (1997). Materiales para una teoría de las identidades sociales. Frontera Norte, 9 (18), 9-28. Recuperado de https://fronteranorte.colef.mx/index.php/fronteranorte/article/ viewFile/1441/891 
Estudios Pedagógicos XLVII N 3: 359-378, 2021

ROL DE LOS APODERADOS Y POLÍTICA DE PARTICIPACIÓN DEL MINISTERIO DE EDUCACIÓN 2002-2016. EL CASO DE DOS ESTABLECIMIENTOS EDUCACIONALES DE UNA COMUNA DE LA REGIÓN DE LOS RÍOS

Gubbins, V. (2012). Familia y Escuela: Tensiones, Reflexiones y Propuestas. Docencia, (46), 6473. Recuperado de https://veronicagubbins.cl/wp-content/uploads/2007/11/Familia-y-Escuelapublicada-rev-docencia-junio-012-1.pdf

Gurdián-Fernández, A. (2007). El Paradigma Cualitativo en la Investigación Socio-Educativa. San José de Costa Rica: Coordinación Educativa y Cultural Centroamericana y Agencia Española de Cooperación Internacional.

Jadue, G. (1997). Factores Ambientales que afectan el Rendimiento Escolar de los Niños provenientes de Familias de Bajo Nivel Socioeconómico y Cultural. Estudios Pedagógicos, (23), 75-80. https://dx.doi.org/10.4067/S0718-07051997000100007

Jiménez de la Jara, M. (2005). Participación Social en Chile. Resultados de la Encuesta CASEN 2003. Departamento de Estudios, División Social, MIDEPLAN.

Kvale, S. (2011). Las entrevistas en Investigación Cualitativa. Madrid: Ediciones Morata, S. L.

Latorre, A. del Rincón, D. y Arnal, J. (1996). Bases Metodológicas de la Investigación Educativa. Barcelona: Hurtado Ediciones.

Lehmann, C. (1994). El Sentido de Pertenencia como Catalizador de una Educación de Calidad. Estudios Públicos, (56), 141-162. Recuperado de https://www.cepchile.cl/cep/estudiospublicos/n-31-a-la-60/estudios-publicos-n-56/el-sentido-de-pertenencia-como-catalizador-deuna-educacion-de-calidad

Martínez-Otero, V. ( 2006). Consideraciones pedagógicas sobre la comunidad educativa: el paradigma de la "escuela-educadora". Revista Complutense de Educación, 17(1), 51-64. Recuperado de http://revistas.ucm.es/index.php/RCED/article/view/RCED0606120051A/15865

Maso, V. y Terrazas, W. (2016). Teorías subjetivas de los apoderados de un colegio particular subvencionado de Antofagasta respecto a su participación en el ámbito académico de sus hijos. Estudios Pedagógicos, 42(2), 227-245. https://doi.org/10.4067/S0718-07052016000200013

Ministerio de Educación. (2002). Política de Participación de Padres, Madres y Apoderados/as en el Sistema Educativo. Santiago de Chile: División de Educación General, Unidad de Apoyo a la Transversalidad. Recuperado de https://www.educarchile.cl/politica-de-participacion-depadres-madres-y-apoderadosas-en-el-sistema-educativo

(2009). DFL $\mathrm{n}^{\circ}$ 2. Fija texto refundido, coordinado y sistematizado de la ley $n^{\circ} 20.370$ con las normas no derogadas del decreto con fuerza de ley $n^{o} 1$, de 2005. [Ley General de Educación]. Recuperado de https://www.leychile.cl/Navegar?idNorma=1014974

(2016). Ley 20.911. Crea el plan de formación ciudadana para los establecimientos educacionales reconocidos por el Estado. Recuperado de https://www.leychile.cl/ Navegar?idNorma $=1088963$

. (2017). Política de Participación de las Familias y la Comunidad en Instituciones Educativas. Santiago de Chile: División de Educación General. Recuperado de http://basica.mineduc.cl/wpcontent/uploads/sites/25/2017/04/Pol\%C3\%ADtica-de-Participaci\%C3\%B3n-de-la-Familia-yla-Comunidad-en-instituciones-educativas.pdf

. (2019). Bases curriculares $3^{\circ}$ y $4^{\circ}$ medio. Recuperado de https://www.curriculumnacional. cl/614/articles-133992_recurso_10.pdf

Ministerio de Desarrollo Social (2018). CASEN 2015. Redes y Cohesión Social Síntesis de Resultados. Recuperado de http://observatorio.ministeriodesarrollosocial.gob.cl/storage/docs/casen/2015/ CASEN_2015_Resultados_Redes_Cohesion_Social.pdf

Ministerio Secretaría General de Gobierno. (2011). Ley 20.500. Sobre Asociaciones y Participación Ciudadana en la Gestión Pública. Recuperado de https://www.ben.cl/leychile/ navegar?idNorma $=1023143$

Navarro, G. Pérez, C. González, A. Mora, O. y Jiménez, J. (2005). Comportamiento Socialmente Responsable en Profesores y Facilitación de la Participación de los Apoderados en el Proceso Enseñanza-Aprendizaje. Psykhe, 14 (2), 43-54. https://dx.doi.org/10.4067/S071822282005000200004 
Organización para la Cooperación y el Desarrollo Económicos (OCDE). (2017). Chile. Scan report on the citizen participation in the constitutional process. OECD Public Governance Reviews. Recuperado de https://www.oecd.org/gov/public-governance-review-chile-2017.pdf

Petrelli, L. (2008). Las Presencias de los Padres en las Instituciones Educativas y la Configuración del Trabajo Docente: El caso de una Escuela Privada de la Ciudad de Buenos Aires. Intersecciones en Antropología, (9), 291-306. Recuperado de http://www.redalyc.org/pdf/1795/179514533021. pdf

Programa de las Naciones Unidas para el Desarrollo (PNUD). (2014). Auditoría a la Democracia. Más y mejor democracia para un Chile inclusivo. Santiago de Chile: PNUD.

Recuperado de https://www.cl.undp.org/content/chile/es/home/library/democratic_governance/ auditoria-a-la-democracia.html

Rivera, M. y Milicic, N. (2006). Alianza Familia-Escuela: Percepciones, Creencias, Expectativas y Aspiraciones de Padres y Profesores de Enseñanza General Básica. Psykhe, 15 (1), 119-135. https://dx.doi.org/10.4067/S0718-22282006000100010

Rodríguez, E. (1994). Criterios de Análisis de la Calidad en el Sistema Escolar y sus Dimensiones. Revista Iberoamericana de Educación. (5), 45-65. Recuperado de https://rieoei.org/historico/ oeivirt/rie05a02.pdf

Rodríguez, G. Gil, J. y García, E. (1999). Metodología de la Investigación Cualitativa. $2^{\circ}$ edición. Málaga: Ediciones Aljibe, S. L.

Stevenson, D. y Baker, D. (1987). The Family-School Relation and the Child's School Performance. Child Development, 58(5) 1348-1357. https://doi.org/10.2307/1130626

Taylor S. J. y Bodgan, R. (1987). Introducción a los métodos cualitativos de investigación. Barcelona: Ediciones Paidós. 